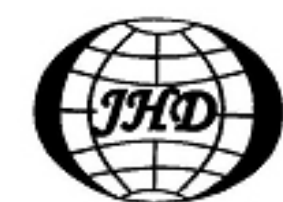

www.sciencedirect.com/ science/journal/10016058

\title{
THE CHARACTERISTICS AND ESTIMATION OF FLOW THROUGH A SINGLE ROUGH-WALLED FRACTURE*
}

\author{
LIU Qing-quan, FAN Hong-guang \\ Laboratory of Environmental Mechanics, Institute of Mechanics, Chinese Academy of Sciences, Beijing 100190, \\ China, E-mail: qqliu @imech.ac.cn
}

(Received October 29, 2011, Revised March 6, 2012)

\begin{abstract}
The flow through a single fracture is numerically studied by means of the Fluent Software. The results show that the roughness of the fracture significantly affects the hydraulic conductivity in the fracture as compared with the cubic law model widely used to describe the flow between two smooth parallel plates. A new model is proposed in this paper, the non-symmetric sinusoidal fracture model, to simulate the flow in a real fracture. This model involves two sinusoidal-varying walls with different phases to replace the flat planes in the cubic law model. The relationships between the effective hydraulic apertures and the phase retardation for different relative amplitudes and wavelengths are numerically investigated. A simple expression of the effective hydraulic aperture of the fracture is obtained, together with the law of the effective hydraulic aperture against the amplitude, the phase retardation and the wavelength of two sinusoidal-varying walls.
\end{abstract}

Key words: fracture seepage, cubic law, effective hydraulic aperture, non-symmetric sinusoidal fracture

\section{Introduction}

Landslide is a kind of natural disasters commonly related with severe damages. Due to fractures widely existing in geologic bodies, the water seepage through fractures is commonly known as one of the major triggers for landslide failures. In fact, the fracturedominated flow is important in many areas, such as in naturally fractured petroleum reservoirs, radioactive waste storages, hydropower projects and geological media. In general, the flow in these media usually takes place through a network of fractures ${ }^{[1,2]}$. The flow through a single fracture is a key to model the transport phenomena in fractured geological media. Therefore, many studies were focused on the single fracture seepage. It is shown that three aspects of seepage researches in a single fracture are involved as follows ${ }^{[3]}$ : (1) the behavior and the velocity distribu-

* Project supported by the National Natural Science Foundation of China (Grant No. 10932012), the National Natural Science Funds of China for Distinguished Young Scholar (Grant No. 10825211) and the National Key Basic Research Program of China (973 Program, Grant No. 2010CB731506). Biography: LIU Qing-quan (1965-), Male, Ph. D., Professor tion of the fluid flow in a single fracture ${ }^{[4,5]}$, (2) the main factors controlling the fluid flow in a single fracture, including stress, aperture, roughness, filling material, multi-field coupling and so on ${ }^{[6,7]}$, (3) the fracture flow numerical simulations ${ }^{[8,9]}$. Various related phenomena were considered, such as anisotropy, contact area, scaling laws, stress effects, and inertial flow effects. The present paper makes no claims of presenting a unified model for all aspects of the single fracture flow, instead, it is our aim merely to develop a model that physically captures more geometric characteristics of a rough-walled fracture as compared with previous geometrical models.

In the earliest time, Lomize, Romm and Louis experimentally investigated the flow through a fracture between a pair of smooth parallel plates. Their experimental results indicate that the flow rate through a fracture is proportional to the cube of the joint aperture, known as the cubic law. Because the fracture walls are considered as a pair of smooth parallel plates, the model is named the parallel plate model. This model is widely used in engineering practice because the cubic law is simple and convenient for applications. Natural joints, however, usually have rough walls, asperity areas, and finite extensions. For large apertures, fracture roughness can be neglected, and a 
parallel plate model is a fairly good approximation. When the roughness is of the same order of magnitude as the mean aperture, the effect of the wall roughness and the change of the fracture aperture due to the seepage cannot be ignored. Experimental observations show that the flow through a fracture decreases its speed more rapidly than that predicted by the cubic law of the mean aperture. It means that the cubic law overestimates the flow in a real fracture with irregular rough surfaces. Therefore, the deviation of a real joint from the ideal conditions has made it necessary to modify the cubic law by introducing the concept of the equivalent hydraulic aperture ${ }^{[10,11]}$, or in other similar ways.

On the basis of the concept of the equivalent hydraulic aperture and experimental obsviations, some empirical relationships, analytical methods and numerical simulations were proposed to consider the influences of fracture aperture and roughness on the hydraulic conductivity. Jiang et al. ${ }^{[12]}$ developed an empirical relationship between surface roughness and mechanical properities for rock joints. According to experimental data, Qian et al. ${ }^{[13]}$ indicated that the average flow velocity in a fracture could be approximated by an empirical exponential function of the hydraulic gradient and the power index in the exponential function. Konzuk and Kueper ${ }^{[14]}$ evaluated and examined the application of the cubic law for the flow through a rough-walled fracture. Sun and Zhao ${ }^{[15]}$, Larsson et al. ${ }^{[16]}$ studied the influences of fracture aperture and roughness on the hydraulic conductivity in the fractured rock mass.

In the other aspect, in order to study the fluid flow in fractures theoretically, the mathematical description of the flow in the fractures should be based on the Navier-Stokes equation ${ }^{[17]}$. Attempts to solve the two-dimensional Navier-Stokes equation analytically were made for simple fracture walls ${ }^{[18-20]}$. Because of the complexity of the surface of the natural fracture and the changeability of the aperture, it is not easy to obtain a simple law by the direct analysis. Instead, the natural fracture is reduced to a geometrical model reflecting some main characteristics of the fracture. One of the most representative models is the sinusoidal fracture model in which the profile of the fracture walls varies sinusoidally. Based on the Reynolds equation, Zimmerman et al. ${ }^{[18]}$ obtained an analytical solution of the equivalent hydraulic aperture for symmetrical sinusoidal seepages. Sisavath et al. ${ }^{[20]}$ used the perturbation theory and the creeping assumption to correlate the mean pressure drop with the mean flow rate and to make the estimation of the conductance or transmissivity of a fracture. A simple expression relating the effective hydraulic aperture with the mean aperture and with the amplitude and the wavelength of the sinusoidal wall profiles is obtained.

Clearly, these empirical and analytical methods can capture some of the effect on hydraulic conductivity caused by the wall-roughness and mechanical aperture. However, they are still limited in applications because the walls of the real fractures are very complex. Therefore, the object of this study is to further examine the influences of the fracture aperture and the roughness on the fracture flow based on the cubic law. A new model called the non-symmetric sinusoidal fracture model is developed by adding the phase retardation to the symmetric sinusoidal fracture. And by means of numerical simulations, a fitting formula of the equivalent hydraulic aperture is obtained.

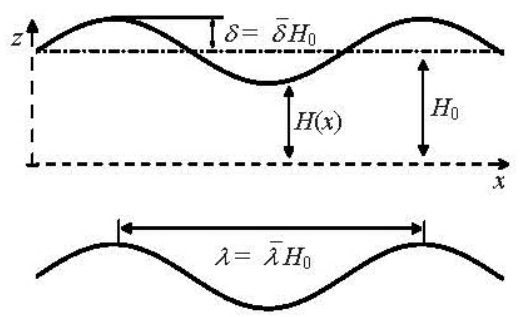

(a) Parallel sinusoidal model

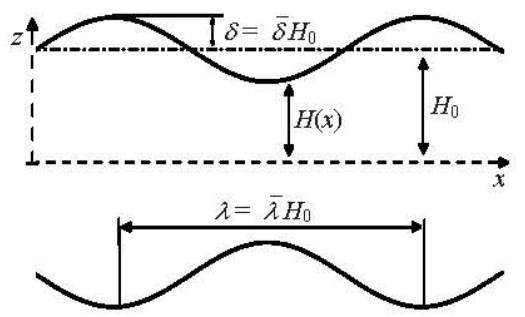

(b) Symmetrical sinusoidal model

Fig.1 Simplified sinusoidal fracture models

\section{Influences of fracture roughness and aperture on the hydraulic aperture}

The roughness and the aperture of a fracture are the most important factors governing the fluid flow through the fracture. Many experimental observations show that the flow through a actual fracture does not follow accurately the cubic law with respect to the mean mechanical aperture. The cubic law often overestimates the flow in a real fracture. In order to obtain an in-depth understanding of the fluid flow in rock fractures, the creeping flow through a fracture is numerically simulated in this paper. For convenience, the sinusoidal fracture model is selected. Although this is not an accurate representation of any specific actual fracture, it does capture some important features of real fracture profiles. Figure 1 shows two kinds of sinusoidal fracture models: (1) a parallel sinusoidal model, and (2) a symmetrical sinusoidal model. $2 H_{0}$ is the mechanical aperture of the fracture, which is defined as the average point-to-point distance between two fracture surfaces, $\delta$ and $\lambda$ are the magnitude and the wavelength of the sinusoidal wave, respe- 
ctively. $\bar{\delta}$ and $\bar{\lambda}$ are the normalized quantities.

The numerical calculations were carried out using the Fluent Software. The equivalent hydraulic aperture, $h_{H}$, was calculated for two kinds of sinusoidal fracture models with the following amplitudes and wavelengths: $\bar{\delta}=0.2,0.4$ and 0.6 , and $\bar{\lambda}=2,4,6,8$ and 10 . For analyzing the effects of the fracture roughness and the aperture on the fracture flow, the relationships between $h_{H}^{3} /\left(2 H_{0}\right)^{3}$ and $\lambda / 2 H_{0}$ are plotted in Fig.2, with comparisons between the equivalent hydraulic aperture and the mechanical aperture for different $\delta$ and $\lambda$.

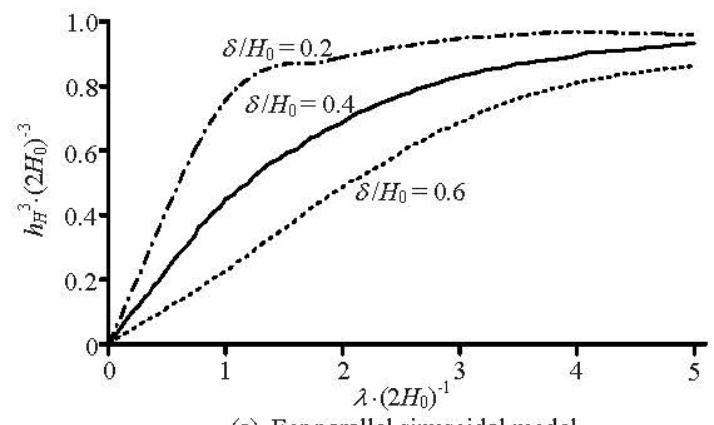

(a) For parallel sinusoidal model

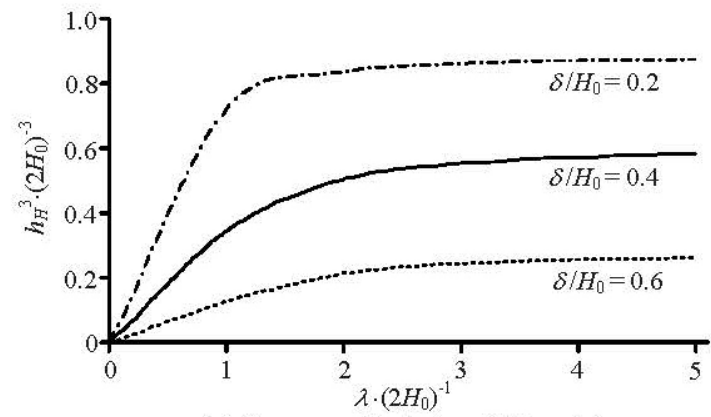

(b) For symmetrical sinusoidal model

Fig.2 Relation between hydraulic and mechanical aperture for different magnitudes and wavelengths of the sinusoidal wave (which represents the different fracture roughnesses and apertures)

The results show that the hydraulic aperture decreases dramatically with the increase of the relative magnitude and the decrease of the wavelength. Namely, the hydraulic aperture decreases as the roughness increases and the mechanical aperture decreases. Obviously, the mechanical aperture is usually larger in magnitude than the hydraulic aperture due to the roughness of the fracture in the rock joints. It means that the cabic law related to the mechanical aperture remarkably overestimates the flow in a natural rough-walled fracture, even for the parallel sinusoidal fracture model. The deviation increases with the increase of the relative magnitude and the decrease of the wavelength.

Based on the symmetrical sinusoidal fracture model, the equivalent hydraulic apertures are also cal- culated for different Reynolds numbers. For constant values of $\bar{\delta}$ and $\bar{\lambda}$, the hydraluic aperture against the flow Reynolds number is shown in Fig.3. The result indicates that the hydraulic aperture decreases with the increase of the Reynolds number and that the cubic law is applicable only for low Reynolds number flows in fractures.

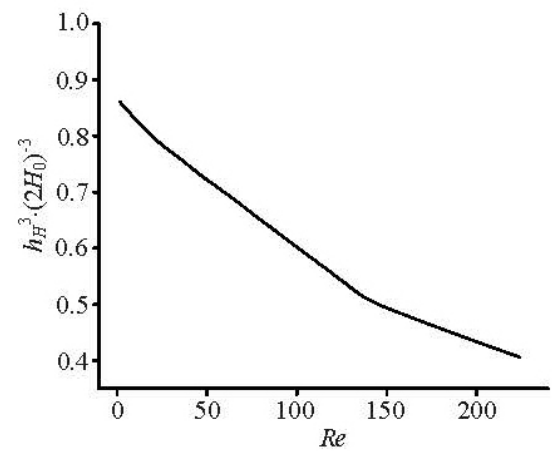

Fig.3 Relation of hydraulic and mechanical apertures vs. Re

\section{Unsymmetrical sinusoidal model}

\subsection{A new geometrical model for rough-walled fra- cture}

As a kind of simplified geometrical models reflecting the main characteristics of the natural fracture, the sinusoidal fracture model is the most representative one. Generally, it includes two kinds of forms: the parallel sinusoidal model and the symmetrical sinusoidal model. Though this model is more realistic than the parallel plate model, it is not realistic enough in some applications in view of the very complex boundaries of a natural fracture. Therefore, in this paper, a new model called the non-symmetric sinusoidal fracture model is proposed by adding the phase retardation to the symmetric sinusoidal fracture. Figure 4 shows the geometrical pattern of the fracture described by the unsymmetrical sinusoidal model.

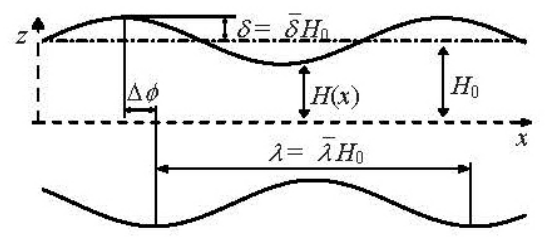

Fig.4 Unsymmetrical sinusoidal fracture model

The expressions of the top and bottom walls of the fracture are as follows:

For the top wall of fracture

$H(x)=H_{0}\left[1+\frac{\delta}{H_{0}} \sin \left(\frac{2 \pi x}{\lambda}\right)\right]$ 
For the bottom wall of fracture

$$
H(x)=-H_{0}\left[1+\frac{\delta}{H_{0}} \sin \left(\frac{2 \pi x}{\lambda}+\Delta \phi\right)\right]
$$

where $H_{0}$ is the mean half-aperture of the fracture, $\delta, \lambda$, and $\Delta \varphi$ are the magnitude, the wavelength and the phase retardation of the sinusoidal wave, respectively. This model can better reflect the main characteristics of the real fracture than the symmetrical and parallel ones.

\subsection{Theoretical analysis}

The laminar, steady, incompressible flow in the fracture is governed by the Navier-Stokes equation:

$$
\frac{\partial u}{\partial x}+\frac{\partial w}{\partial z}=0
$$

$u \frac{\partial u}{\partial x}+w \frac{\partial u}{\partial z}=-g \frac{\partial h}{\partial x}+v\left(\frac{\partial^{2} u}{\partial x^{2}}+\frac{\partial^{2} u}{\partial z^{2}}\right)$

$u \frac{\partial w}{\partial x}+w \frac{\partial w}{\partial z}=-g \frac{\partial h}{\partial z}+v\left(\frac{\partial^{2} w}{\partial x^{2}}+\frac{\partial^{2} w}{\partial z^{2}}\right)$

where $u$ and $w$ are the velocity components in $x$ and $z$ directions, respectively, $v$ is the viscosity coefficient of water, $g$ is the gravity acceleration, $h$ is the total hydraulic head. The $x$ direction is defined as the flow direction in the fracture and the $z$ direction is vertical to the $x$ direction.

For a slow flow, the velocity gradient in the flow direction is very small and can be ignored when the change of the fracture aperture is smooth. Then Eqs.(4) and (5) can be simplified into the following forms:

$$
\begin{aligned}
& g \frac{\partial h}{\partial x}=v \frac{\partial^{2} u}{\partial z^{2}} \\
& g \frac{\partial h}{\partial z}=v \frac{\partial^{2} w}{\partial z^{2}}
\end{aligned}
$$

Integrating above equations and combining with the continuity Eq.(3), we obtain the following equation, i.e., the Reynolds equation

$$
\nabla\left[H^{3}(x, \delta, \lambda) \nabla h\right]=0
$$

where $H(x, \delta, \lambda)$ is the function of the fracture aperture.

As the pressure condition is known and the velo- city is unknown for the fracture in the experiments, it is reasonable to use the pressure boundary conditions here:

$x=0, h=h_{l}$

$x=l, h=h_{r}$

where $l$ is the fracture length, $h_{l}$ and $h_{r}$ are the heights in the entrance and in the exit, respectively. In order to keep the cubic law, the equivalent hydraulic aperture is introduced here. For the same pressure difference $\Delta p$ and the flow rate $Q$, the distance $h_{H}$ between the two parallel plates of length $l$ is defined as the equivalent hydraulic aperture.

Based on the Reynolds equation, the analytical solution of the equivalent hydraulic aperture for a symmetrical sinusoidal seepage is as follows ${ }^{[17,18]}$

$h_{H}^{3}=\frac{\left(2 H_{0}\right)^{3}\left(1-\bar{\delta}^{2}\right)^{5 / 2}}{1+\left(\frac{\bar{\delta}^{2}}{2}\right)}$

Considering the effect of the wavelength and the amplitude of the fracture walls, Sisavath et al. ${ }^{[20]}$ obtained the second-order perturbation solution of the equivalent hydraulic aperture for the symmetrical sinusoidal fracture model by using the Stokes equations

$h_{H}^{3}=\frac{\left(2 H_{0}\right)^{3}\left(1-\bar{\delta}^{2}\right)^{5 / 2}}{1+\frac{\bar{\delta}^{2}}{2}}\left[1+\frac{\bar{\delta}^{2}}{\bar{\lambda}^{2}} \frac{36}{15} \frac{\pi^{2}\left(1-\bar{\delta}^{2}\right)}{1+\frac{\bar{\delta}^{2}}{2}}\right]^{-1}$

where $\bar{\lambda}$ is the normalized wavelength of the top and the bottom boundaries, $\bar{\lambda}=\lambda / H_{0}$ is the half of the average distance between the top and the bottom boundaries in the symmetrical fracture, $\bar{\delta}$ is the normalized magnitude, $\bar{\delta}=\delta / H_{0}, h_{H}$ is the equivalent hydraulic aperture.

For the unsymmetrical sinusoidal fracture walls, we introduce a function of the phase retardation in the formula (12). When the Reynolds number is low and the change of the fracture wall is smooth, the equivalent hydraulic aperture can be given as in Eq.(13) for the unsymmetrical sinusoidal fracture model.

$$
h_{H}=f(\bar{\lambda}, \bar{\delta}) g(\Delta \varphi)
$$


where

$$
f(\bar{\lambda}, \bar{\delta})=\frac{\left(2 H_{0}\right)^{3}\left(1-\bar{\delta}^{2}\right)^{5 / 2}}{1+\frac{\bar{\delta}^{2}}{2}}\left[1+\frac{\bar{\delta}^{2}}{\bar{\lambda}^{2}} \frac{36}{15} \frac{\pi^{2}\left(1-\bar{\delta}^{2}\right)}{1+\frac{\bar{\delta}^{2}}{2}}\right]^{-1},
$$

$g(\Delta \varphi)$ is the function of the phase retardation $\Delta \varphi$.

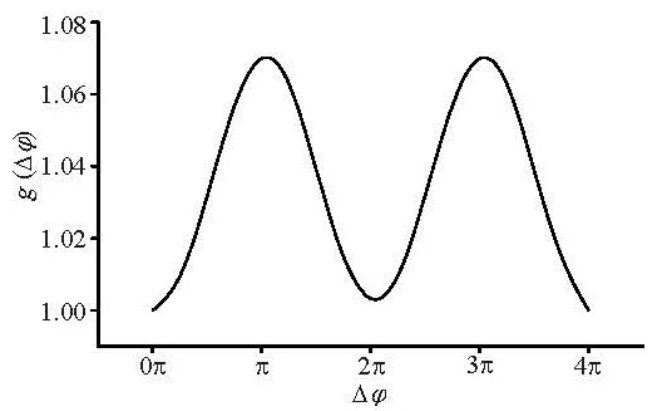

Fig.5 The variation of function $g(\Delta \varphi)$

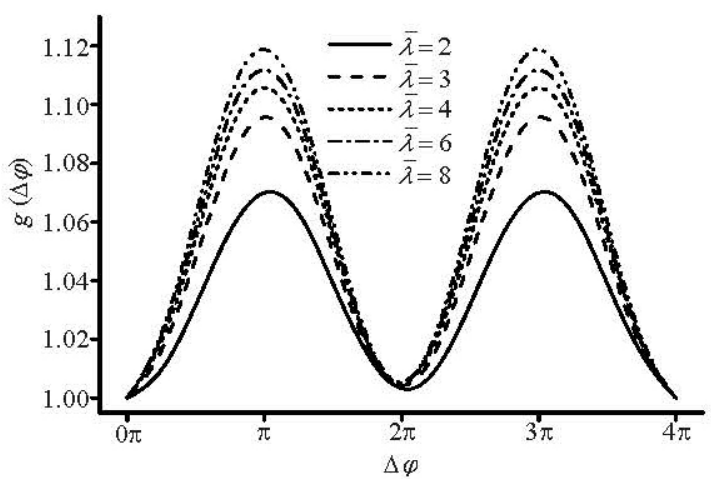

(a) $\bar{\delta}=0.2$

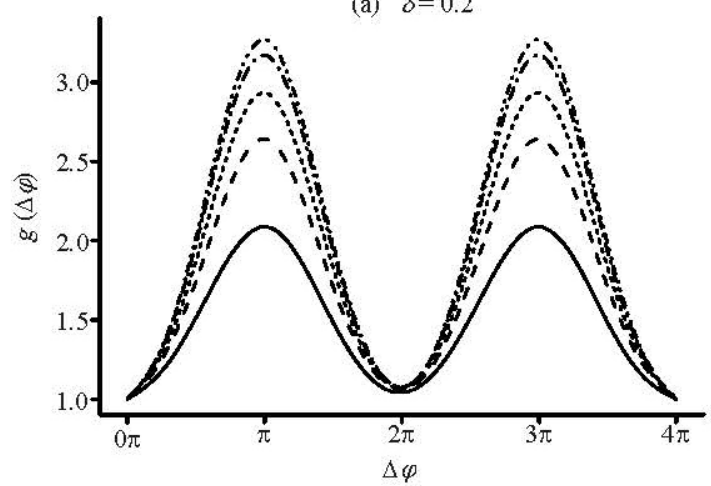

(b) $\bar{\delta}=0.6$

Fig.6 The variation of function $g(\Delta \varphi)$ for different $\bar{\delta}$ and $\bar{\lambda}$

With the seepage model of a single fracture, the determination of the equivalent hydraulic aperture is the key if the cubic law is still used in the new model. As the boundaries of the unsymmetrical sinusoidal fracture are complex and there are many parameters in the flow equations, it is difficult to find an analytical solution for the equivalent hydraulic aperture. Instead, the numerical simulation is employed here and the equivalent hydraulic aperture is obtained by fitting of the numerical results based on the formula (13).

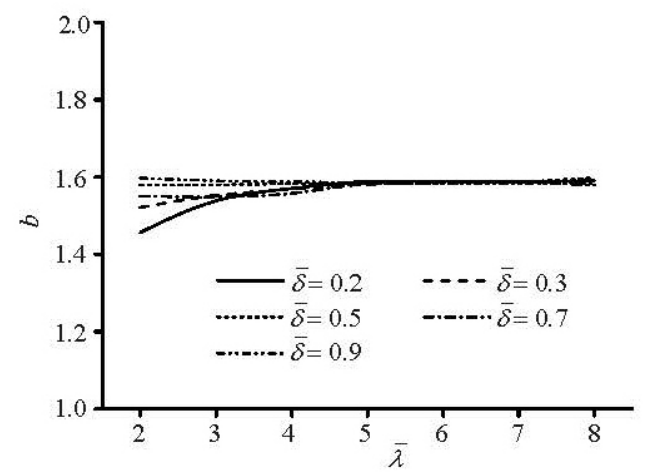

Fig.7 Relationship between parameter $b$ and wavelength

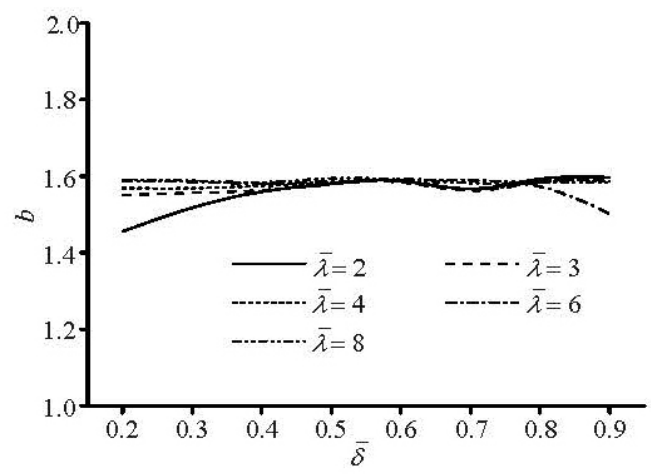

Fig.8 Relationship between parameter $b$ and magnitude

\section{Numerical simulation and analysis}

By means of the Fluent Software, the flows in a fracture with different $\delta$ and $\lambda$ are simulated for different phase retardations $\Delta \varphi$. From the numerical results, $g(\Delta \varphi)$ can be obtained as shown in Fig.5, in which $\Delta \varphi$ ranges from 0 to $4 \pi$ and $\bar{\delta}=\delta / H_{0}=$ $0.2, \bar{\lambda}=\lambda / H_{0}=2$. Actually, the geometrical pattern of the fracture walls in Fig.6 shows that when $\Delta \varphi=0$, it is degenerated to the symmetrical sinusoidal fracture model, that means: $g(0)=1, g(\Delta \varphi)=$ $g(\Delta \varphi+2 \pi)$. Consequently, based on the results shown in Fig.5, we can approximately assume that $g(\Delta \varphi)$ takes a sinusoidal form. Then we have

$g(\Delta \varphi)=1+a[\sin (\Delta \varphi+b)-\sin b]$

In order to verify the assumption and get the values of $a$ and $b$ in Eq.(14), Fluent software is used for simulating the fracture flow in the low Reynolds number condition. In the simulations, $\bar{\delta}$ ranges from 0.2 to 0.9 with interval $0.1, \bar{\lambda}$ ranges from 2 to 8 with interval 1 , and $\Delta \varphi$ ranges from 0 to 
Table 1 Values of $a$ for different wavelengths and magnitudes

\begin{tabular}{cccccccc}
\hline $\bar{\delta}$ & \multicolumn{7}{c}{$\lambda$} \\
\cline { 2 - 7 } & 2 & 3 & 4 & 5 & 6 & 7 & 8 \\
\hline 0.2 & -0.0372 & -0.0507 & -0.0558 & -0.0581 & -0.0594 & -0.062 & -0.0628 \\
0.3 & -0.0868 & -0.1247 & -0.1405 & -0.1483 & -0.151 & -0.1517 & -0.1536 \\
0.4 & -0.1682 & -0.2473 & -0.2861 & -0.3027 & -0.3137 & -0.3165 & -0.321 \\
0.5 & -0.3138 & -0.4687 & -0.534 & -0.5766 & -0.6017 & -0.6145 & -0.6375 \\
0.6 & -0.5729 & -0.8598 & -1.015 & -1.095 & -1.145 & -1.176 & -1.198 \\
0.7 & -1.134 & -1.736 & -2.094 & -2.298 & -2.421 & -2.504 & -2.548 \\
0.8 & -2.738 & -4.342 & -5.375 & -6.058 & -6.452 & -6.7 & -6.872 \\
0.9 & -11.82 & -20.12 & -26.43 & -30.34 & -32.58 & -34.64 & -35.67 \\
\hline
\end{tabular}

$4 \pi$ with interval $0.25 \pi$. Flow rates $q$ in the fracture can be obtained for a given pressure difference $\Delta p$ and different values of $\bar{\delta}, \bar{\lambda}$ and $\nabla \varphi$. Therefore, the equivalent hydraulic aperture $h_{H}$ can be obtained according to the cubic law. And $g(\Delta \varphi)$ can be calculated from Eq.(13). Then the values of $a$ and $b$ in Eq.(14) can be obtained by fitting Eq.(13). Figure 6 shows the variation of function $g(\Delta \varphi)$ for different wavelengths and magnitudes of the sinusoidal function wall. The results show that $g(\Delta \varphi)$ does behave like a sinusoidal function although with different parameters for different $\bar{\delta}$ and $\bar{\lambda}$. Figures 7 and 8 show the fitted results of the parameter $b$ against the wavelength and the magnitude of the sinusoidal function wall.

Based on the results shown in the Figures 7 and 8, we can see that $b$ is equal to $\pi / 2$ approximately. Substituting $b=\pi / 2$ into Eq.(14), the equivalent hydraulic aperture for the unsymmetrical sinusoidal fracture model takes the following form

$$
\begin{gathered}
h_{H}^{3}=\frac{\left(2 H_{0}\right)^{3}\left(1-\bar{\delta}^{2}\right)^{5 / 2}}{1+\frac{\bar{\delta}^{2}}{2}}\left[1+\frac{\bar{\delta}^{2}}{\bar{\lambda}^{2}} \frac{36}{15} \frac{\pi^{2}\left(1-\bar{\delta}^{2}\right)}{1+\frac{\bar{\delta}^{2}}{2}}\right]^{-1} \\
\{1+a[\cos (\Delta \phi)-1]\}
\end{gathered}
$$

where $a$ is related to the wavelength and the magnitude and is given in Table 1.

The results in Table 1 show that $a$ is not a constant but a function of $\bar{\lambda}$ and $\bar{\delta}$. Assuming that the relation of $a$ with $\bar{\lambda}$ and $\bar{\delta}$ is in the form,

$$
a=k_{1} \bar{\delta}^{k 2} \sqrt{1+\frac{k_{3}}{\bar{\lambda}+k_{4}}}
$$

we can obtain the fitted parameters $k_{1}=-207.5443$, $k_{2}=-13.0044, k_{3}=-7.2663, k_{4}=5.6400$.

The equivalent hydraulic aperture of the unsymmetrical sinusoidal fracture seepage is then

$$
\begin{gathered}
h_{H}^{3}=\frac{\left(2 H_{0}\right)^{3}\left(1-\bar{\delta}^{2}\right)^{5 / 2}}{1+\frac{\bar{\delta}^{2}}{2}}\left[1+\frac{\bar{\delta}^{2}}{\bar{\lambda}^{2}} \frac{36}{15} \frac{\pi^{2}\left(1-\bar{\delta}^{2}\right)}{1+\frac{\bar{\delta}^{2}}{2}}\right]^{-1} \\
\left\{1+k_{1} \bar{\delta}^{k 2} \sqrt{1+\frac{k_{3}}{\bar{\lambda}+k_{4}}}[\cos (\Delta \phi)-1]\right\}
\end{gathered}
$$

The Reynolds number used in the simulation in this paper is smaller than 10, so Eq.(17) is applicable under the conditions of $\bar{\lambda}>2 \quad \bar{\delta} \leq 0.9$ and $R e \leq$ 10 .

\section{Discussions}

An approximate expression of the equivalent hydraulic aperture is derived to describe the flow through a fracture with sinusoidally-varying walls. This solution improves the results obtained by using the symmetrical sinusoidal model, by accounting for the effect of the phase retardation between two sinusoidally-varying walls. In Eq.(17), the third term in the bracket represents the effect of the phase retardation between two walls, that is missing in the symmetrical sinusoidal model. Obviously, the approximation obtained in this paper provides a substantial improvement over the perturbation solution derived by Sisavath et al. ${ }^{[20]}$ on the basis of the symmetrical sinusoidal model. In fact, when the phase retardation $\Delta \varphi$ goes to zero formula (17) will be degenerated to the the perturbation solution derived by Sisavath, when $\bar{\lambda}$ goes to infinity, formula (17) will be degenerated 
to Eq.(11) (derived by Zimmerman et al. ${ }^{[18]}$ ), and when $\bar{\delta}$ goes to zero, it will be degenerated to the cubic law.

In order to verify Eq.(17), numerical calculations were carried out using the Fluent Software. The equivalent hydraulic aperture was calculated for two sets of amplitudes and wavelengths: $\bar{\delta}=0.2, \bar{\lambda}=2$ and $\bar{\delta}=0.8, \bar{\lambda}=4$ and with following phase retardations: $\Delta \phi=0, \pi / 4, \pi / 2,3 \pi / 4, \pi, 5 \pi / 4,3 \pi / 2$, $7 \pi / 4$ and $2 \pi$. Three wavelengths (unit cells) were used for each simulation. It is shown that the flow becomes fully-developed by the third wavelength. Good agreement is obtained between the numerical results and the approximation solution (17) (see Fig.9).

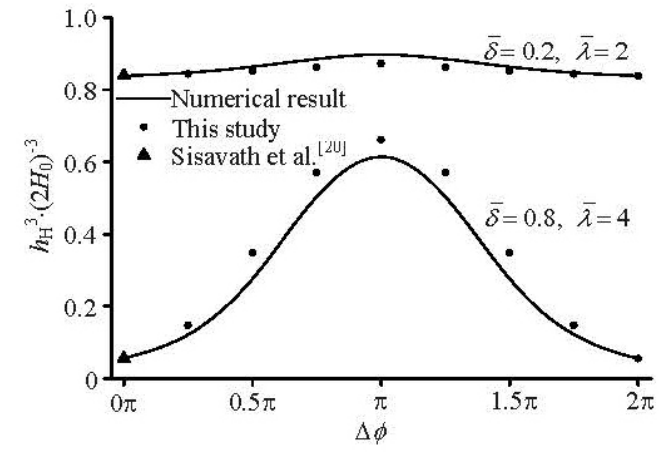

Fig.9 Comparison of the results obtained with the approximation solution (Eq.(17) ) The symbols $\circ$ correspond to the the perturbation solution (Eq.(12)) by Sisavath et al. ${ }^{[20]}$

The symmetrical sinusoidal model does not consider the phase retardation between two sinusoidallyvarying walls, and consequently, the perturbation solution's range of acceptable accuracy is restricted to small phase retardations. Nevertheless, the perturbation solution provides a useful lower bound, which is the value obtained as $\Delta \phi \rightarrow 0$. Figure 9 also shows that when the amplitude is small, the effect of the phase retardation between two walls is small and negligible. It means that the perturbation solution based on the symmetrical sinusoidal model is in the range of acceptable accuracy for a small phase retardation between two walls. However, when the phase retardation between two walls increases, its effect must be considered as done in this paper. The simulated results also indicate that the flow velocity in the fracture decreases with the increases of the amplitude and the frequency of the sinusoidal walls of the fracture. For a fixed amplitude and frequency, the velocity takes the minimum value when the top and bottom walls are fully symmetrical, and the maximum value in the case of fully consistent top and bottom walls.

\section{Conclusions}

The flow through a single fracture is studied by numerical simulations. The following conclusions are drawn from this study:

(1) The roughness and the aperture of a fracture are the most important factors governing the fluid flow through the fracture. The hydraulic aperture based on the cubic law, in general, is not equal to the mechanical aperture for rough-walled fractures. The mechanical aperture is usually larger in magnitude than the hydraulic aperture due to the roughness of the fracture. Generally, the hydraulic aperture decreases dramatically as the roughness increases and the mechanical aperture decreases.

(2) A new geometric model called the non-symmetric sinusoidal fracture model is developed to describe the flow in a rough-walled fracture. By adding the phase retardation to the symmetric sinusoidal fracture, this model can reflect the main characteristic of the real fracture much better than the symmetrical model.

(3) On the basis of the concept of the equivalent hydraulic aperture and the cubic law, a expression relating the effective hydraulic aperture of the fracture to the mean aperture and to the amplitude, the wavelength and the phase of the sinusoidal wall profiles is obtained through numerical simulations.

(4) Very good agreement is obtained between the numerical results and the solution of the model proposed in this paper. The approximation in this paper provides a substantial improvement over the perturbation solution derived by Sisavath et al. on the basis of symmetrical sinusoidal model. This result also demonstrates that the new model extends the application range of the cubic law to more general fractures with complex geometry boundaries.

\section{References}

[1] CHAI Jun-rui,WU Yan-qing. Analysis of 3-D seepage field through main fracture network in dam foundation[J]. Journal of Hydrodynanics, Ser. A, 2003, 18(4): 459-462(in Chinese).

[2] HE Yang, CHAI Jun-rui and TANG Zhi-li et al. Numerical analysis of 3-D unsteady seepage through fracture network in rock mass[J]. Journal of Hydrodynamics, Ser. A, 2007, 22(3): 338-344(in Chinese).

[3] XIONG Xiang-bin, ZHANG Chu-han and WANG Enzhi. A review of steady state seepage in a single fracture of rock[J]. Chinese Journal of Rock Mechanics and Engineering, 2009, 28(9): 1839-1846(in Chinese).

[4] XU Guang-xiang, ZHANG Yong-xing and HA Qiu-ling. Super-cubic and sub-cubic law of rough fracture seepage and its experiments study[J]. Journal of Hydraulic Engineering, 2003, (3): 74-79(in Chinese). 
[5] BAGHBANAN A., JING L. R. Hydraulic properties of fractured rock masses with correlated fracture length and aperture[J]. International Journal of Rock Mechanics and Mining Sciences, 2007, 44(5): 704719 .

[6] WANG Yuan. Coupling characteristics of stress and fluid flow within a single fracture[J]. Chinese Journal of Rock Mechanics and Engineering, 2002, 21(1): 8387(in Chinese).

[7] GRASSELLIA G., EGGER P. Constitutive law for the shear strength of rock joints based on three-dimensional surface parameters $[\mathrm{J}]$. International Journal of Rock Mechanics and Mining Sciences, 2003, 40(1): 25-40.

[8] JEONG W. A numerical study on flow and transport in a rough fracture with self-affine fractal variable apertures[J]. Energy Sources, 2008, 30(7): 606-619.

[9] KOYAMA T., LI B. and JIANG Y. et al. Numerical modelling of fluid flow tests in a rock fracture with a special algorithm for contact areas[J]. Computers and Geotechnics, 2009, 36(1/2): 291-303.

[10] OLSSON R., BARTON N. An improved model for hydromechanical coupling during shearing of rock joints $[\mathrm{J}]$. International Journal of Rock Mechanics and Mining Sciences, 2001, 38(3): 317-329.

[11] WANG Yuan, SU Bao-yu. Research on the behavior of fluid flow in a single fracture and its equivalent hydraulic aperture $[\mathrm{J}]$. Advances in Water Science, 2002, 13(2): 61-68(in Chinese).

[12] JIANG Y., LI B. and TANABASHI Y. Estimating the relation between surface roughness and mechanical properities of rock joints[J]. International Journal of Rock Mechanics and Mining Sciences, 2006, 43(6): 837-846.
[13] QIAN J. Z., ZHAN H. B. and ZHAO W. D. et al. Experimental study of turbulent unconfined groundwater flow in a single fracture[J]. Journal of Hydrology, 2005, 311(1-4): 134-142.

[14] KONZUK J. S., KUEPER B. H. Evaluation of cubic law based models describing single-phase flow through a rough-walled fracture[J]. Water Resources Research, 2004, 40: W02402.

[15] SUN J. P., ZHAO Z. Y. Influences of fracture aperture and roughness on hydraulic conductivity in fractured rock mass[C]. New Trends in Fluid Mechanics Research-Proceeding of the Sixth International Conference on Fluid Mechanics. Guangzhou, 2011, 372-374.

[16] LARSSON M., NIEMI A. and TSANG C. F. A study of flow-wetted surface area in a single fracture as a function of its hydraulic conductivity distribution[J]. Water Resources Research, 2012, 48: W01508.

[17] YEO I. W., GE S. M. Applicable range of the Reynolds equation for fluid flow in a rock fracture $[\mathrm{J}]$. Geosciences Journal, 2005, 19(4): 347-352.

[18] ZIMMERMAN R. W., KUMAR S. and BODVARSSON G. S. Lubrication theory aanalysis of the permeability of rough-walled fractures[J]. International Journal of Rock Mechanics and Mining Sciences, 1991, 28(4): 325-331.

[19] BASHA H. A., EL-ASMAR W. The fracture flow equation and its perturbation solution[J]. Water Resources Research, 2003, 39(12): 1365-1379.

[20] SISAVATH S., AL-YAARUBI A. and PAIN C. C. et al A simple model for deviations from the cubic law for a fracture undergoing dilation or closure[J]. Pure Applied Geophysics, 2003, 160(5-6): 1009-1020. 